\title{
Le syndrome Micromégas
}

Les glissières du rapport nature/culture : l'exemple du cannibalisme

\section{Georges Guille-Escuret}

\section{OpenEdition}

Journals

Édition électronique

URL : https://journals.openedition.org/tc/3949

DOI : $10.4000 /$ tc.3949

ISSN : 1952-420X

Éditeur

Éditions de l'EHESS

\section{Édition imprimée}

Date de publication : 31 décembre 2008

Pagination : 182-205

ISSN : 0248-6016

\section{Référence électronique}

Georges Guille-Escuret, «Le syndrome Micromégas », Techniques \& Culture [En ligne], 50 | 2008, mis en ligne le 31 décembre 2010, consulté le 29 septembre 2022. URL : http://journals.openedition.org/tc/ 3949 ; DOI : https://doi.org/10.4000/tc.3949 


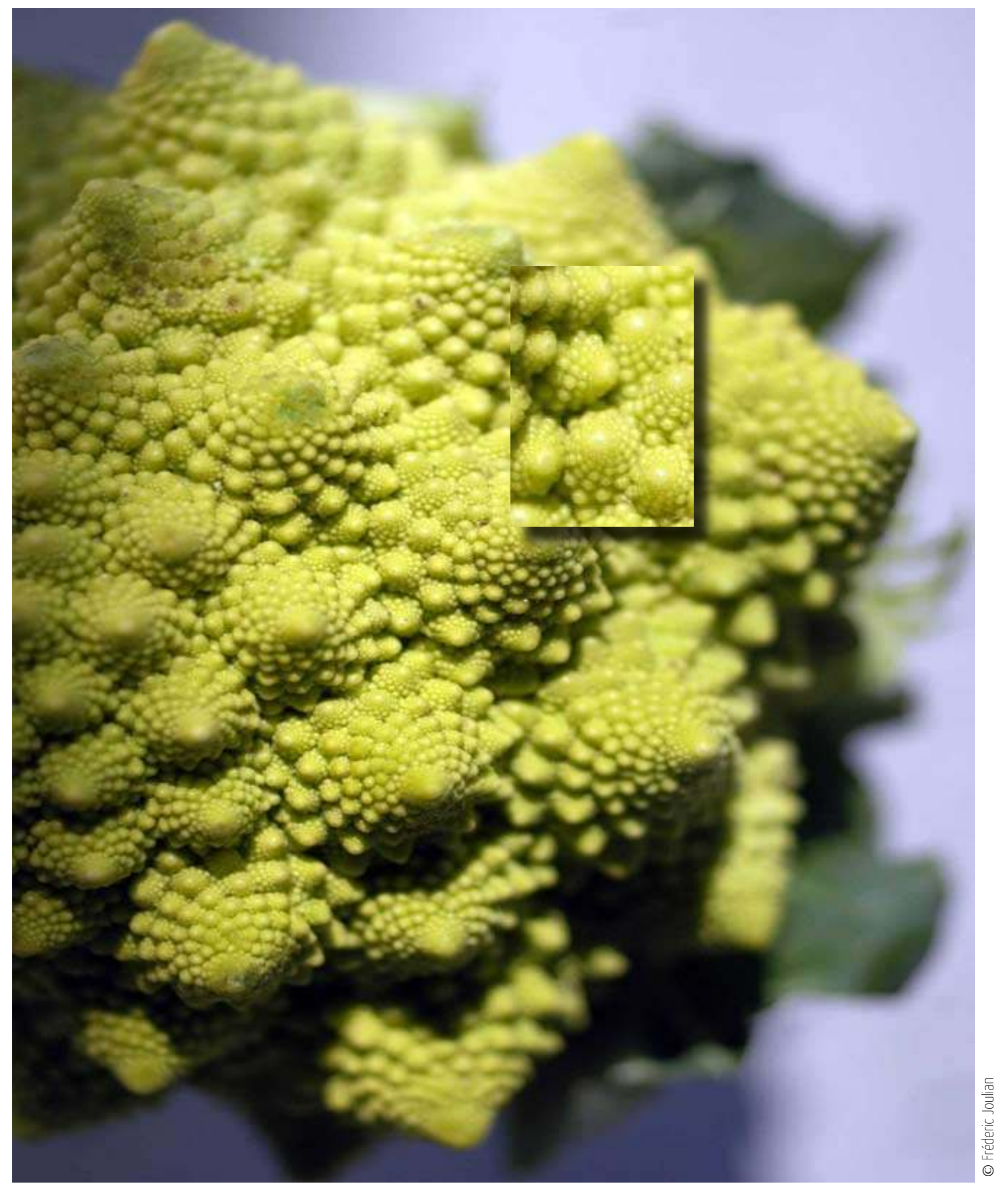




\title{
LE SYNDROME MICROMÉGAS
}

\section{Les glissières du rapport nature/culture L'exemple du cannibalisme}

\author{
Le cannibalisme, de par la phobie qu'il inspire à notre civilisation, augmente les \\ risques de dérapages théoriques et offre ainsi une sorte de loupe méthodologique \\ devant le problème de la biologisation du social.
}

Est-il légitime de transformer les leçons d'un événement anecdotique en un fait d'évolution, ou de transférer la conclusion d'une observation ponctuelle dans la longue durée d'un vaste monde? Sûrement pas. Pourtant, ces extensions purement analogiques sont fréquentes en sciences naturelles et en sciences humaines et cela d'autant plus que la proposition est « téléportée » des unes vers les autres. Tout ce qui tourne autour du rapport nature/ culture, de la frontière animal/homme, ou de la connexion biologique/social se caractérise comme une zone de stagnation théorique: non seulement les antagonismes philosophiques semblent s'y cristalliser désespérément, mais ils paraissent eux-mêmes produire une immobilisation des tendances intellectuelles et forger des structures de pensée capable de résister à l'afflux des informations pourtant inattendues que livre régulièrement l'observation scientifique. Les cibles de la recherche ont beau avoir beaucoup changé depuis le milieu du XIX ${ }^{e}$ siècle, ainsi que les supports de l'argumentation, force est de constater deux faits troublants au sujet des discussions majeures qui se sont nouées sur ces sujets: elles s'agglutinent irrésistiblement les unes aux autres jusqu'à ce que leur confusion totale fasse figure d'évidence et 
elles se répètent périodiquement sous des rénovations sémantiques superficielles. Ces deux volets - confusion et répétition - ne sont ici distingués que par commodité, car un seul ressort autorise l'extension thématique et la reproduction historique: une liberté tacitement et collectivement accordée au glissement analogique des raisonnements.

Est-ce une cause ou un effet? Cette mystérieuse plasticité des dissensions théoriques ne se borne pas à garantir la pérennité des grandes alternatives philosophiques, elle permet également la reconduction de contradictions que les institutions rendent plus palpables, à commencer par la tension «traditionnelle » qui sévit entre les tenants des sciences de la vie et ceux des sciences sociales. Par exemple, côté « confusion », la synonymie souvent affirmée ex nihilo entre les rapports nature/culture et inné/acquis facilitent d'emblée un ordre de marche de toute la biologie à la rencontre des phénomènes sociaux: peu importerait que l'explication fondatrice adressée à la sociologie soit d'ordre génétique, éthologique ou écologique (malgré les profondes divergences épistémologiques qui séparent ces secteurs), du moment qu'elle puisse se réclamer d'une autorité de principe des sciences de la vie sur les sciences sociales, affreusement « molles » comme chacun sait. Et, du côté de la «structure réitérative des grandes idéologies parascientifiques » (Tort 1983), on se contentera de rappeler le réaménagement complet du vieux darwinisme social en sociobiologie, moyennant un remplacement du support imaginé pour l'évolution de l'altruisme: l'hérédité lamarckienne des caractères acquis a cédé la place aux spéculations du gène égoïste. Là aussi, la résurrection a été secondée par une réorganisation pluridisciplinaire du réductionnisme (absorption supposée de l'éthologie par la zone d'influence de la dynamique des populations, assujettissement de fait de la socio-écologie animale à une « écologie comportementale » créée pour l'occasion): une restriction terrible des possibilités de dialogue avec les anthropologues qui ne pouvaient accepter que la discussion soit subordonnée à la reconnaissance d'une loi universelle jaillie du monde moléculaire.

\section{Prenez un certain nombre de généticiens...}

Quelle est l'efficacité de ces expédients et pourquoi s'y complairait-on? Les « évidences à géométrie variable » sont assurément redoutables par les flous qu'elles protègent. Elles le sont davantage encore par leur puissante adaptabilité. À l'inverse de ce que l'on pourrait soupçonner a priori, en effet, il est rare que leur efficacité suprême réside dans un arrogant refus de recevoir la réfutation, ou de prendre acte d'une réflexion critique. Préparées à l'esquive, elles développent plutôt une admirable virtuosité à enregistrer et à digérer l'objection censée les anéantir de manière à se rétablir ensuite dans leur plénitude initiale, moyennant quelques concessions insignifiantes.

L'anecdote qui suit fournira une illustration assez éloquente d'un blocage subconscient parmi les membres d'une spécialité éminente. Elle concerne un débat qui eut lieu en 1997, lors d'un congrès pluridisciplinaire 
consacré à l'actualité du darwinisme. Une communication avait été présentée qui prenait appui sur l'utilisation récente, par une flopée de biologistes renommés, d'une célèbre allégorie, attribuée au mathématicien Émile Borel, au sujet du hasard: prenez un certain nombre de singes, mettez-les devant un certain nombre de machines à écrire et, au bout d'un certain temps, l'un d'entre eux tapera Hamlet sans une faute.

À l'encontre des spéculations supputant les chances de voir une « sélection cumulative » réaliser l'événement, sinon sur la pièce en entier, du moins sur un vers (Dawkins 1989), on montra que l'expérience avait bel et bien été produite, en " grandeur nature » qui plus est, et que ses résultats sont approximativement connus. Il suffit en effet de se souvenir que, Shakespeare et les imprimeurs sont des Primates et qu'ils appartiennent à l'ensemble défini par la fable: dès lors, la difficulté n'est plus de situer le moment où le miracle se produit, mais celui où apparaissent les singes. Ironie beaucoup moins dérisoire qu'il n'y paraît au premier regard, car elle dénonce l'irrationalité des savants calculs qui prétendent mesurer, isolément et à long terme, les probabilités de sélection d'un trait comportemental ou physiologique, en supposant silencieusement la clause d'un « toutes choses égales par ailleurs ». Un avantage adaptatif n'existe que dans un contexte précis et l'évolution, justement, est intimement associée au fait que, dans la nature, les choses ne demeurent jamais longtemps « égales par ailleurs » : Hamlet a été correctement tapé à la machine par des singes, mais la grandeur de l'aléatoire avait quitté la lice depuis longtemps (Guille-Escuret 1997). Autrement dit, le hasard est certainement une machine active et puissante à l'échelle du microcosme, mais il est canalisé et orienté par des systèmes d'interaction exerçant leurs contraintes à l'échelle du macrocosme.

L'exposé provoqua quatre interventions successives, émanant toutes de généticiens se déclarant favorables à cette contestation, car hostiles à la récupération de la fable de Borel dans une perspective d'évolution biologique. Le malentendu vient immédiatement après: tous les quatre voulurent « compléter » l'argumentation en ajoutant des objections purement techniques sur le traitement du hasard en lui-même. Quatre fois, il fut expliqué que ce renfort, malgré sa bienveillance, n'était en l'occurrence nullement souhaitable parce qu'il ramenait la discussion dans le microcosme, alors que la controverse portait sur l'extension illégitime du micro (l'image rêvée d'un champ parsemé de bêtes poilues et de claviers) vers le méga (une évolution où les singes qui pianotent sur la machine ont, entre autres choses, abandonné beaucoup de leur système pileux et se dandinent sur deux pieds).

En pure perte: les participants issus de la biologie moléculaire retinrent exclusivement le caractère globalement abusif de la fable du singe dactylographe. Un réflexe commun les a conduits à rechercher une formulation subsidiaire qui contournerait l'enjeu embarrassant, c'est-à-dire les relations entre la compétence de la causalité génétique et celle de la synthèse écologique (et/ou sociologique), de telle sorte que la première pourrait continuer à proposer des intuitions sur les adaptations à long terme sans demander à la seconde d'évaluer la fiabilité de ces convictions. 


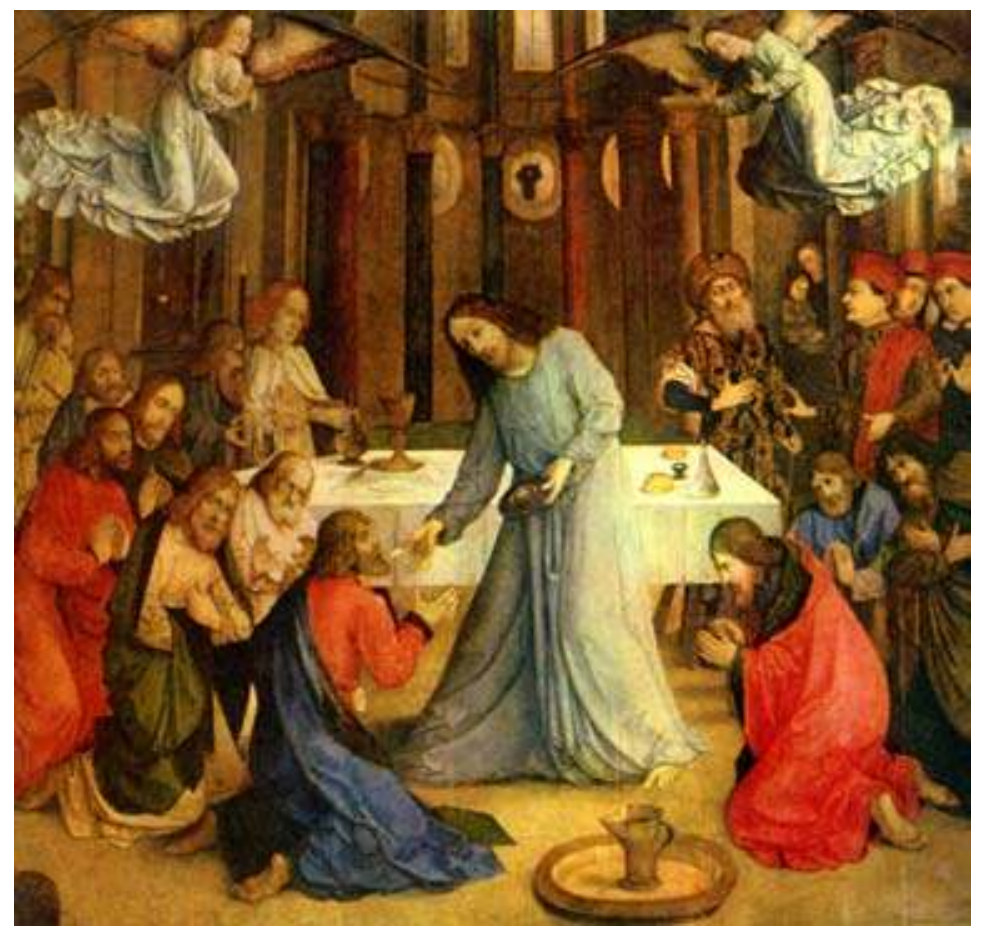

\section{« Institution de l'Eucharistie »}

José Just de Gand: Communion des apôtres - Urbin, Galerie Nationale.

\section{Eucharistie et Saturne}

Deux forces s'entrelacent dans la répulsion que le cannibalisme inspire à la civilisation occidentale: la première relève du politique, la seconde du sacré. Ainsi, pour les Grecs, la culture est impensable tant que règne « l'allèlophagie » (littéralement, la consommation de l'autre): Zeus, lui-même, instaure un nouvel ordre après avoir échappé, grâce à une ruse de sa mère, à la voracité de son père, Chronos. Voltaire s'inscrit dans ce sillage quand il ironise sur les difficultés de la vie sociale quand on s'entre-dévore. Avec la chrétienté, s'ajoute un autre aspect: la valeur religieuse du corps humain rend l'absorption de chair humaine d'autant plus sacrilège qu'un des moments clefs de la liturgie catholique, la communion, implique une ambivalence sur ce plan. La réception de l'eucharistie oscille entre théophagie et anthropophagie, mais, curieusement, les commentaires à ce sujet semblent se croire obligés de choisir entre l'une des deux versions.

D’une façon générale, les réactions scandalisées des Européens face à des « sociétés cannibales » présentent de multiples combinaisons à partir de ces deux aversions classiques qui, cependant, tendent au XIX siècle, à chercher une synthèse dans un rejet « humaniste ».

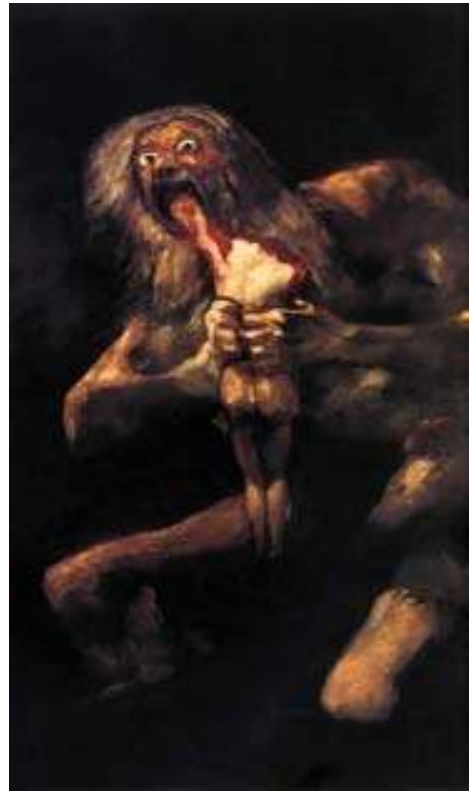

« Saturne dévorant ses enfants » Francisco de Goya: Musée du Prado. 


\section{L'analogie, zoom de la croyance: bref rappel sur la sociobiologie}

Or, cette sorte d'aveuglement n'est pas moins régulier dans la construction des théories que dans leur assimilation et il touche plus souvent les spécialistes de sciences habituées à présenter des causalités que les tenants des disciplines où l'on s'attache à modéliser des systèmes et des structures: autour de la démarcation nature/culture, la transposition d'une observation ponctuelle et momentanée à la longue durée d'un vaste monde, ou, au contraire, la reconnaissance d'une loi évolutive dans un incident repéré deux ou trois fois en un lieu donné se répètent à l'envi non seulement dans l'élaboration des explications scientifiques mais aussi dans les refus que celles-ci suscitent.

D'autre part, il faut noter qu'une constante stratégique exerce ici une action logiquement transversale : le discours positif, la « proposition » au sens premier, est l'apanage des analyses causales (telles que celles habituellement produites par la génétique et l'éthologie), tandis que les points de vue de l'écologie ou de l'ethnologie, se retrouvent nécessairement dans des " positions de contre », ou de challenger, puisqu'il ne saurait y avoir de cause éminente à un système ou à une structure. L'écologue et le sociologue non réductionnistes sont en somme dans l'incapacité de "rendre les coups » en produisant une contre-proposition satisfaisante.

La sociobiologie moderne et ses dérivés offrent une mine inépuisable de ces « téléportations » de la réflexion où la magie le dispute à la science-fiction: chaque fois, l'analogie se substitue au travail contrôlable de la comparaison, alors même que celui-ci serait réalisable. Parmi les plus belles pépites du genre, rappelons la thèse de la naissance du social chez les insectes, avec la prépondérance « statistique » des espèces haplodiploïdes $^{1}$ (femelles provenant d'œufs fécondés, mâles ayant deux fois moins de chromosomes): "l'eusocialité », ou socialité complète serait apparue dix ou douze fois chez les hyménoptères, et seulement deux fois ailleurs, chez les isoptères (termites) et les hominidés. Les statistiques ont bon dos et leur autorité y est parfaitement illusoire (Guille-Escuret 1996)² , l'affirmation reposant en réalité sur toute une série de transferts opportunistes entre le niveau de la superfamille (fourmis), l'ordre (hyménoptères), la classe (insectes) et le règne (animal). Quand une information contraire surgit, telle que l'ordre des termites (où toutes les espèces sont diploïdes et sociales), le raisonnement « saute » dans un cadre où l'exception sera contenue, puis dans un autre où l'on pourra carrément l'oublier. Il existe une vision concurrente de la naissance du social chez les insectes dont la priorité a été totalement ignorée grâce à ces expédients: tous les insectes « eusociaux» ont des mandibules broyeuses qui leur permettent de construire des habitats collectifs et de vivre dans des espaces confinés où la sélection des phéromones liées au comportement profite de conditions particulières (Guille-Escuret 1997b). 
Linterprétation des infanticides chez l'entelle (Presbytis entellus, simien asiatique) est une autre merveille: chaque fois qu'une comparaison contrôlable risque d'invalider l'égoïsme du gène, c'est à un autre étage qu'une analogie insaisissable vient éblouir le public: on va chercher au loin une confirmation qui oblitérera un démenti tout proche. Par exemple, il y a de vastes régions où, apparemment, l'entelle ne pratique jamais l'infanticide et où plusieurs mâles adultes cohabitent dans un groupe (Guille-Escuret 1994; Thierry 1997): la même espèce, mais, cette fois, avec des gènes permissifs, voire fraternels. Aussi a-t-on bondi au-dessus de cet écueil pour rapporter aussitôt le corpus isolé des meurtres à quelques données plus ou moins « similaires » recueillies chez les grands félins.

\section{Entelle et son petit}

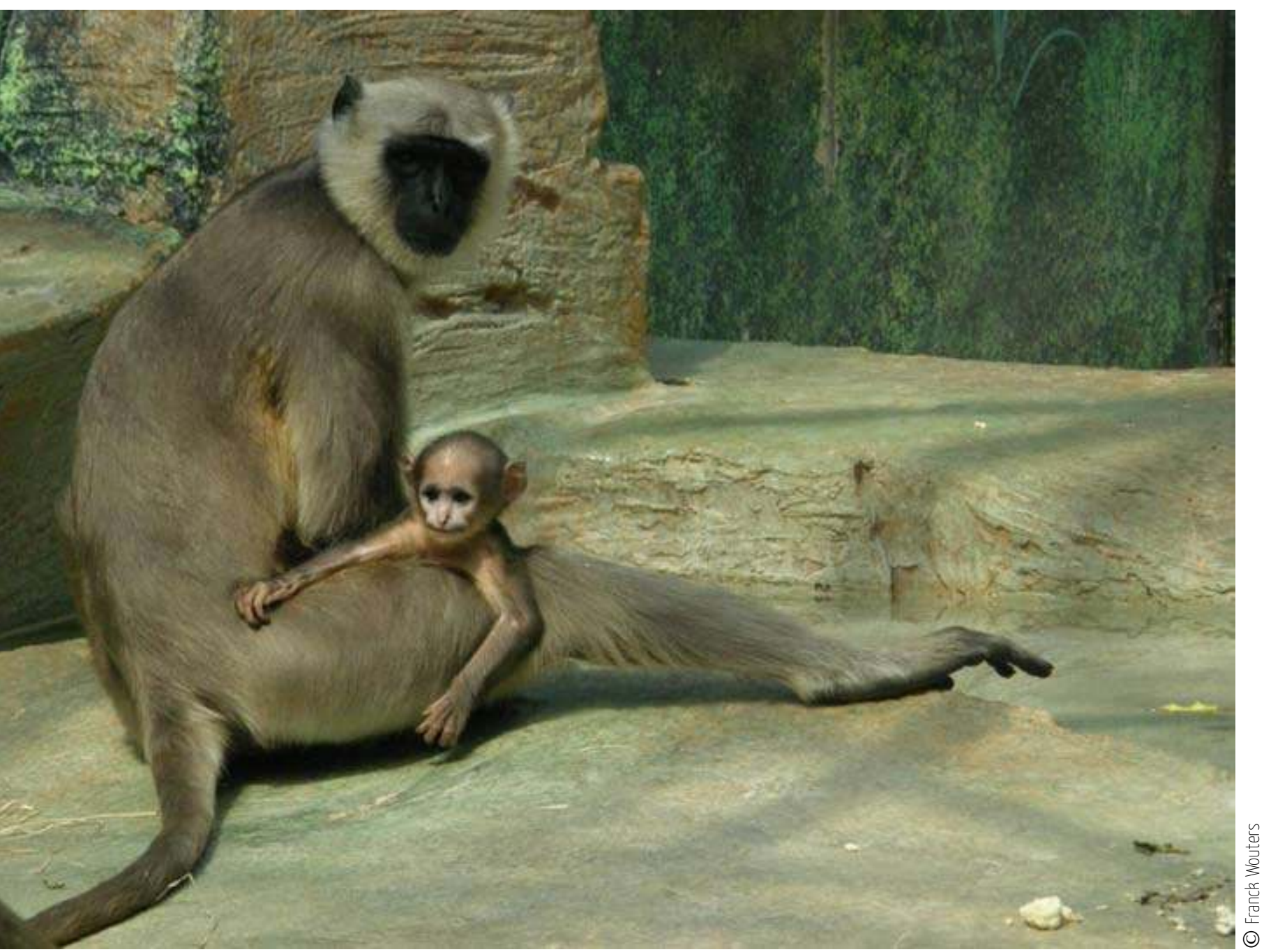


Bien plus récemment, aux États-Unis, une conjecture sur la sélection de groupe d'un trait culturel a été maintes fois saluée pour sa rigueur et sa prudence: elle suppute en effet, en travaillant sur les villages de NouvelleGuinée, qu'une sélection se distinguant de la simple variation individuelle en opérant à l'étage du groupe ne pourrait influencer sensiblement une option culturelle qu'en disposant d'un laps de temps compris entre cinq et dix siècles (Soltis, Boyd \& Richerson 1995). Comme dans la fable de Borel, le calcul fait toutefois une « impasse » fantastique sur la fluctuation des interactions entre les composantes de la culture pendant ces quelques centaines d'années. L'image primitive d'un génome " en sac de haricots » (c'est-à-dire où les gènes s'accumulent sans se lier les uns aux autres) a été victorieusement combattue et elle est aujourd'hui officiellement repoussée: devra-t-on maintenant livrer une bataille homologue devant la conception d'une culture « en sac de haricots »? Une telle problématique gèle - ou, selon un mot à la mode, réifie - simultanément les groupes sociaux et les «éléments » de la culture. Mais c'est précisément la double condition nécessaire au libre passage de l'observation momentanée (d'une communauté isolable sur un territoire délimité) à la tendance millénaire (d'une population humaine aux contours indistincts sur une région floue).

\section{Le cannibalisme: une loupe pour les impairs de la méthode}

Ne nous attardons pas davantage sur la sociobiologie, sous peine d'enfermer la faiblesse visée dans la perversité idéologique d'une école ou d'une orientation théorique, alors que le phénomène dépasse largement ce cadre. Le thème du cannibalisme convient mieux, la fébrilité n'émanant plus de l'angle sous lequel le rapport nature/culture est abordé mais d'une névralgie inhérente à l'ambiguité du sujet lui-même: les registres de l'alimentation, de l'agression, du symbole et du politique ne cessent de s'y entremêler dans des combinaisons opaques, propices à souligner toutes les concurrences imaginables entre l'environnement et l'histoire, ou entre la pulsion et la structure. Nous nous arrêterons ici sur trois moments troublants: le premier en anthropologie biologique (1970-1979), le second en ethnologie (1977-1979) et le troisième en primatologie (1986-1998).

Le glissement du micro vers le méga et son complément, l'affrontement de la cause et du système, sont survenus sous une forme extraordinairement simple quand, après plusieurs décennies d'un silence prude sur ces pratiques trop choquantes (ou trop disposées à raviver les vieux mépris ethnocentristes), une perspective nutritionniste a été brusquement soutenue à propos des cannibalismes mélanésiens. Constatant la pauvreté en protéines animales du milieu, les auteurs se demandaient quelle part pouvait prendre la consommation des ennemis tués dans la satisfaction des besoins alimentaires sur ce plan. 


\section{Protéine animale}

Dans les années 1960, les anthropologues découvrent que les sociétés de Nouvelle-Guinée ont un régime alimentaire extraordinairement végétarien: parfois, la participation de la chair animale y dépasse à peine $1 \%$ de la nourriture. Une hypothèse se répand alors: dans la mesure où les protéines végétales sont moins complètes que les protéines animales, ces dernières seraient absorbées de préférence dans des moments de crise ou de tension sociales. On songe d'abord, aux hécatombes rituelles que subit l'élevage porcin, mais l'idée s'applique ensuite spontanément à l'anthropophagie et elle inspire indubitablement la thèse de Harner sur le cannibalisme aztèque.

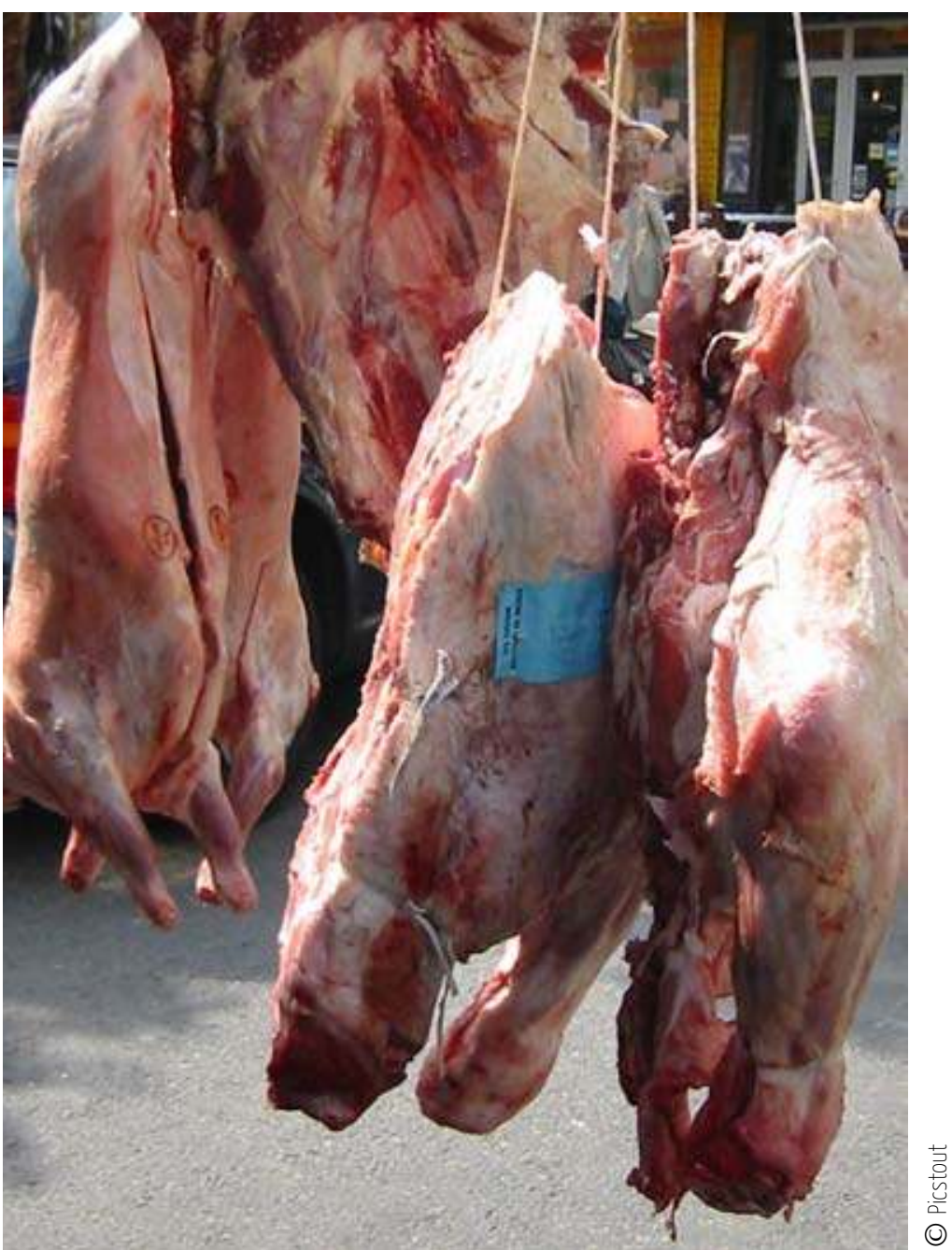


En 1970, une première publication met bizarrement le feu aux poudres en contestant l'intérêt économique de la pratique: Stanley Garn et Walter Block (1970) écrivent qu'un groupe de 60 personnes absorbant un homme de $50 \mathrm{~kg}$ par semaine ne recevrait que $15 \%$ de ses besoins en protéines. Quatre commentaires répondent ${ }^{3}$, dont un joliment fantaisiste. Les autres protestent: primo contre un isolement artificiel de la viande humaine de son contexte (Randall 1971); secundo contre le fait que la situation précaire des groupes concernés n'est pas prise en compte, bien qu'elle favorise des comportements opportunistes (Walens 1971); et, tertio, contre le peu de pertinence d'une telle moyenne. Ce dernier argument, vient d'Andrew Vayda (1970), figure de proue d'une écologie humaine rivée à un fonctionnalisme « dur », et spécialiste des Maori: s'appuyant sur un travail déterminant de Rappaport (1968), il se demande si la pratique irrégulière du cannibalisme n'a pas joué un rôle dans les phases de stress social.

Trois ans plus tard, l'étude est reprise, corrigée et complétée par deux nouveaux auteurs, Dornstreich et Morren: ils établissent que le groupe envisagé plus haut par Garn et Bullock n'aurait pas reçu $15 \%$ des ses besoins mais 35 et $40 \%$. D'autre part, un village d'une centaine de personnes se nourrissant de 5 à 10 êtres humains par an en retirerait une quantité de viande égale à celle que fournissent les porcs. Grosso modo, chaque homme semble représenter $1 \%$ des besoins du hameau. Pour finir, Stanley Garn (1979) renchérira sur sa position initiale, affirmant que non seulement le cannibalisme n'a pas de valeur économique, mais qu'il peut même être à long terme coûteux en calories ${ }^{4}$.

On veut bien le croire, compte tenu de la propension des hommes à être mieux armés que les cochons. Et aussi plus implacablement rancuniers. Dans ces dialogues hallucinants, la rareté des protéines animales, avec ou sans stress social initial, est considérée en tant que cause potentielle d'un comportement sans aucun égard pour son irrationalité socio-écologique flagrante : l'intérêt nutritif de l'homme est comparé à celui du porc puis infiltré dans des scénarios qui impliquent une mortalité régionale annuelle de cinq à dix pour cent dans le meilleur des cas. Autrement formulé: si cette cause-là est véritablement éminente dans le microcosme, la population disparaît en moins d'une génération de la zone étudiée et le macrocosme est irréalisable. En clair, il serait sage d'en déduire que l'espèce humaine n'a pas pu émerger ou qu'elle s'est éteinte au Paléolithique, en raison des probabilités trop grandes qu'avait cette déplorable pulsion d'en ruiner les espérances démographiques. La survie des Papous est également un mystère, à moins que les Occidentaux ne soient arrivés « juste à temps».

Un paramètre a rendu le piège pratiquement inévitable: le fait que l'affaire ait été amorcée par un désir de prouver que, même en acceptant de se situer sur un plan exclusivement nutritif, l'homme n'était pas un gibier utile. Comme pour les généticiens voulant rejeter la fable de Borel pour des motifs « à eux », l'entreprise naissait d'un paradoxe: le congénère est par nature impensable « sur un plan exclusivement nutritif ». 
Nous survolerons plus rapidement le second moment - le débat ethnologique autour du cannibalisme aztèque (Harner 1977; Harris 1979; Sahlins 1979) -, nettement plus connu et dont l'absurdité demeure proche de l'affaire précédente. Historiquement d'abord, car Michael Harner, l'auteur de la thèse selon laquelle l'aristocratie aztèque aurait profité biologiquement de l'anthropophagie en accaparant les victimes, se fonde en partie sur la publication citée plus haut de Dornstreich et Morren (par le biais de la rareté des protéines animales accessibles au Mexique). Épistémologiquement ensuite: son article s'intitule «The ecological basis for Aztec sacrifice », ce qui nous ramène à l'ineptie d'un écosystème (ou d'un système social) forgé autour d'une stimulation centrale. Par définition, un système ne saurait avoir de cause puisqu'il se présente comme un ensemble de rapports qui se stimulent ou s'entretiennent les uns les autres.

Harner, cependant, va encore plus loin dans la prestidigitation théorique et fait jouer l'astuce en sens inverse. En campant officiellement sur le terrain de l'écologie, donc de la biocénose et de la cohérence, il délivre son explication fonctionnelle d'une obligation terrible: éclairer la genèse du phénomène "révélé ». Protégée par le système dont on se souvient soudain qu'il ne repose pas sur une " cause », voilà que la causalité n'a plus besoin d'indiquer son origine.

La durée de l'Empire aztèque ne dépasse guère quatre-vingts ans! Voilà qui semble court pour permettre à la nature de dicter les détails d'une loi inédite au sein de la culture. En outre, au temps où les habitants de Mexico sacrifient leurs prisonniers, l'anthropophagie guerrière sévit dans toute la Mésoamérique, au sud des actuels États-Unis, dans les Caraïbes et en Amérique du Sud. Faut-il ajouter que, sur cette superficie, la rareté des protéines animales, la puissance coercitive d'une classe aristocrate et la pression démographique ne sont nullement des constantes mais des paramètres dont la présence même est variable? Il n'y a aucune commune mesure entre l'extension spatiale et temporelle du cannibalisme américain et celle de la cause découverte par Harner et immédiatement approuvée par Marvin Harris.

Et pourtant, dans sa réplique caustique et mordante, le grand pourfendeur anglo-saxon des simplifications réductionnistes, Marshall Sahlins, ne s'en offusque pas: sa critique adopte le cadre forgé par Harner, alors qu'il serait facile et nettement plus efficace de commencer par en dénoncer les flagrantes insuffisances. Négligence déplorable mais anodine? Peutêtre pas: Sahlins tire de ce contournement un bénéfice qu'il n'hésite pas ensuite à investir dans une contre-attaque. On a vu que ses adversaires ont travesti le terrain de leur analyse pour promouvoir une cause sous couvert d'écologie: en répondant point par point, il admet implicitement la consistance socio-écologique du substrat de la thèse nutritionniste mais, du coup, il prend une revanche appréciable sur le sort qui le contraint d'habitude à subir l'incapacité de la sociologie dans l'art d'établir des causes proprement dites. En d'autres termes, combattre chaque argument 


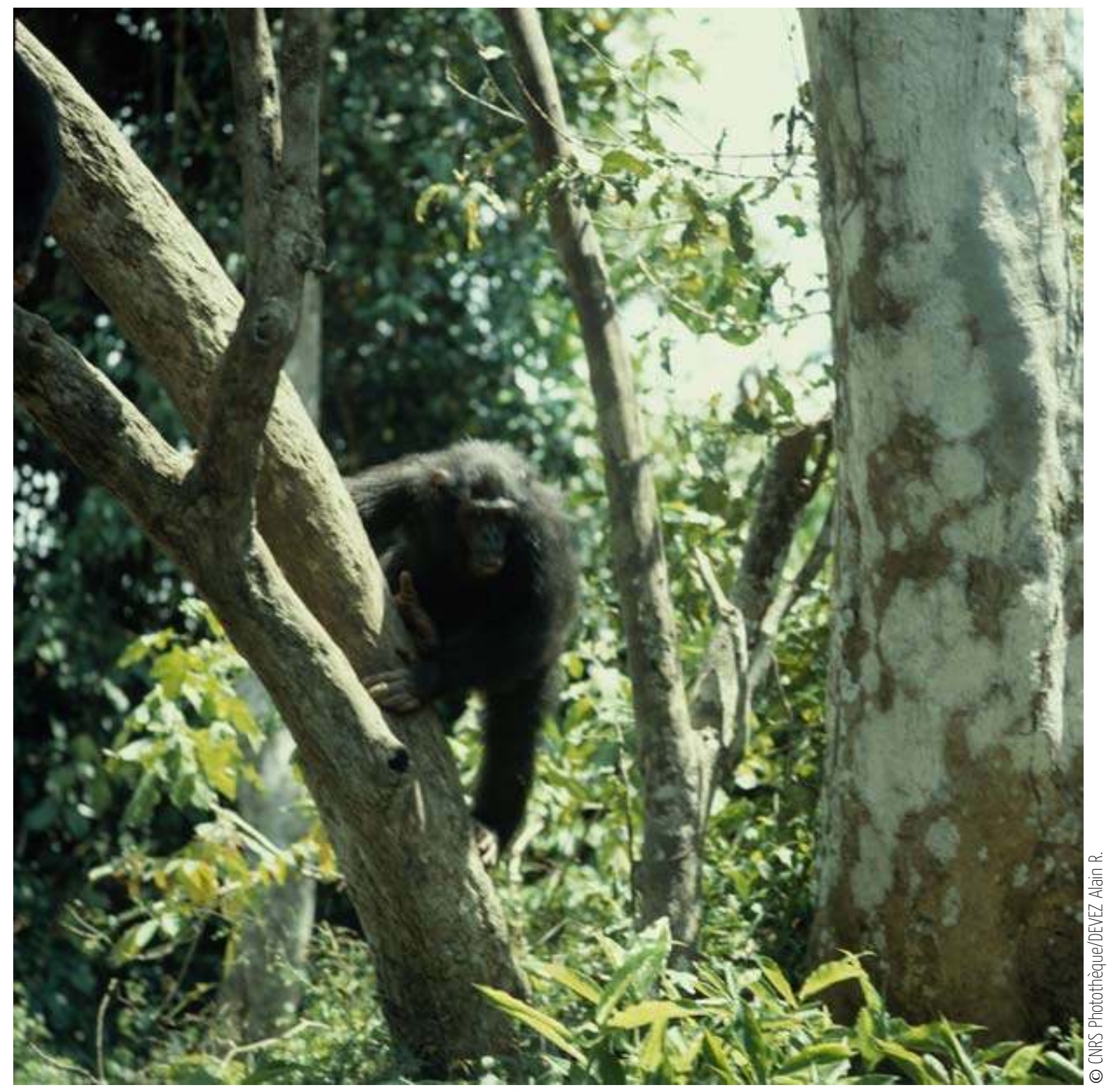

Au regard des sciences naturelles actuelles, les chimpanzés sont devenus nos nouveaux « primitifs ». lls représentent en effet une nouvelle base de référence pour l'étude de l'hominisation. Attractifs en raison de leur proximité biologique vis-à-vis de notre espèce, ils le sont aussi par leurs aptitudes de chasseurs, leur régime omnivore et la découverte d'une forme de diversité proprement culturelle dans leur espèce. Mais le vieux réflexe évolutionniste est

toujours là qui sévertue à traquer chez eux des bribes initiales, des sources ou des morceaux embryonnaires de notre humanité, au lieu de sintéresser prioritairement à leurs stratégies écologiques et à la variabilité de leurs modes de vie, voire à leurs histoires. Les prédations observées sur les enfants sont alors prestement renvoyées au cannibalisme guerrier des Amérindiens ou des Mélanésiens, plutôt que d'être analysées pour leur signification propre. 
de la thèse causale soutenue pas Harner et Harris en entérinant de facto l'entité biogéographique et historique qui la supporte devient pour lui l'opportunité de remplacer une cause inacceptable par une cause infiniment plus crédible. La même qu'il avait dressée (en recourant au même procédé) devant la sociobiologie: la faculté symbolique (concept non sociologique, soit dit en passant). De là, l'alternative fantasmagorique offerte au public: la protéine ou le symbole. Dans ce cas, le «zoom analogique » et la « géométrie variable de l'évidence » ont été utilisés dans les deux sens, d'une part, et par les deux interprétations antagonistes, d'autre part.

Troisième et ultime illustration: les meurtres cannibales chez les chimpanzés, relatés depuis une trentaine d'années. Les spécialistes de l'hominisation les ont vite perçus comme symptomatiques, voire prometteurs d'un éclairage sur l'agressivité des carnivores primitifs qui sommeille plus ou moins jusque chez le civilisé de Wall Street. Certains ont recommandé la prudence (Hublin 1982), mais quelques primatologues ont allègrement replongé dans des extrapolations à la manière de Konrad Lorenz: Jane Goodall (1986) est hélas du nombre.

Résumons les principales données accessibles en cherchant tous les obstacles qui se lèvent devant l'extension du microcosme temporel des chimpanzés au macrocosme paléolithique (et en laissant de côté que les hommes préhistoriques dont on présume qu'ils peuvent avoir été mangés par leurs congénères sont fort rarement de jeunes enfants):

- 13 prédations intraspécifiques ont été notées entre 1967 et 1989, toutes sur des enfants ${ }^{5}$. Le premier exemple a été observé en Ouganda, et les autres, un millier de kilomètres au sud, dans les aires relativement proches ${ }^{6}$ de Gombe Stream et de Mahale Mountains ( 6 chacune). Le score est énorme comparé aux autres espèces qui, hormis le macaque japonais, ne sont toutefois pas aussi constamment épiées.

- 8 de ces événements (soit plus de $61 \%$ ) ont eu lieu dans la double zone de Gombe et Mahale en moins de 4 ans (sur 22), soit d'août 1975 à juin 1979.

- À Gombe, une même femelle, prophétiquement nommée Passion, a commis en moins de 16 mois (août 1975-novembre 1976) 3 meurtres cannibales dans sa propre communauté, alors que les 3 autres cas enregistrés dans cette réserve venaient de mâles attaquant des enfants « étrangers ». La furie de Passion cessa brusquement mais certains mâles la tinrent ensuite à l'écart pendant des années. Goodall, alarmée par cette tuerie, calcula que sur un laps de temps de trois années, un seul enfant avait survécu.

- À Mahale, au contraire, ce sont les mâles qui attaquèrent. Selon Russell H. Tuttle (1986), les infanticides auraient eu lieu dans la zone de chevauchement entre les domaines vitaux de deux groupes, ou à proximité.

- Aucun exemple de cannibalisme ni d'infanticide n'a été répertorié en Afrique de l'Ouest, malgré les longues recherches qui ont été consacrées aux chimpanzés en Guinée et Côte d'Ivoire (Hiraiwa-Hasegawa 1992).

On ne sait plus qui, du psychanalyste ou de l'écologue, doit hériter du dossier. Les pulsions de Passion, le chimpanzé de Gombe qui toilette 
l'enfant qu'il vient de tuer, le mâle dominant de Mahale qui s'insurge contre ces agressions, des signes d'inhibition, tout souligne la complexité du phénomène à l'échelon individuel (Norikoshi 1982). Un film récent montre un mâle de Mahale en train d'agiter frénétiquement un enfant mort au-dessus de la tête de la mère: difficile de penser à ce geste en évitant l'anthropomorphisme de la cruauté (d'après Frédéric Joulian, à propos d'un film NHK).

Moyennant quoi, si flagrantes soient-elles, les stimulations « personnelles » ne règlent pas seules le problème. Les guenons dont l'enfant est capturé sont souvent en position marginale dans le groupe. Goodall souligne deux faits essentiels: quand elles sont sexuellement réceptives, les femelles migrent volontiers vers une autre communauté qu'elles intègrent plus ou moins superficiellement et durablement (l'évitement de l'inceste est général chez les primates). En outre, les relations entre deux groupes résultant d'une scission plus ou moins ancienne sont fébriles dans leurs démonstrations d'affection qui, à tout moment, sont susceptibles de « virer » à l'agressivité. L'éthologie des relations sociales approche là des réseaux d'interactions terriblement subtils qui seront sûrement plus longs à modéliser que les tendances individuelles et qui, par ailleurs, demanderont un éventail d'informations beaucoup plus étendu que celui actuellement présenté par les publications.

Enfin, dans le cadre élargi d'une socio-écologie proprement dite, on en est réduit à de timides conjectures, car le support central manque: modifications des écosystèmes, évaluations de la densité de population, etc. S'il s'agissait d'une autre espèce, on serait assurément tenté d'attribuer le cannibalisme de Gombe-Mahale et son inexistence probable en Afrique occidentale à un ou plusieurs paramètres environnementaux. Mais, depuis que les comparaisons inter-régionales y ont révélé des divergences éthologiques parfaitement compatibles avec la notion ethnologique d'opposition culturelle (McGrew 1992), et que des activités techniques y ont même laissé entrevoir des symptômes de « tradition » (Joulian 1995), l'écologie classique a perdu le monopole sur l'interprétation des divergences et doit apprendre à incorporer dans ses analyses une rémanence inédite des conduites chez l'animal.

Un contraste mérite d'être retenu: on a vu qu'une même femelle de Gombe Stream, bientôt secondée par sa propre fille, était à l'origine de la plupart des meurtres intraspécifiques, alors qu'à Mahale Mountains ceux-ci ont semblé plus nettement liés à un degré de friction entre groupes. Nos « cousins » soulignent ainsi la présence de deux formes radicales d'histoire appelant des cadres de référence incommensurables, avec deux angles différents pour une connexion éventuelle entre histoire et évolution.

La guenon assassine exprime prioritairement les effets d'une histoire « personnelle» qui concerne pour commencer les sciences du psychisme. Le fait que Jane Goodall se soit sentie obligée d'entraver autant que possible les tentatives d'infanticide à Gombe pour préserver l'espérance démographique de la troupe fixe par ailleurs l'enjeu d'une 
réflexion du point de vue évolutif : jusqu'à quel point une population fragilisée par les contraintes d'une reproduction très spécialisée est-elle en mesure de tolérer de pareilles anomalies sans ruiner ses perspectives d'avenir? Ce sont les bénéfices et les coûts de la variabilité individuelle du comportement qui entrent ici en lice: même minoritaires ou sporadiques, de semblables accès de fureur se succédant à un certain rythme hypothéqueraient le destin de l'espèce.

À Mahale, les perspectives socio-écologiques dominent d'emblée, réclamant vite des comparaisons de situations: les cadres de référence du fait sont plus réduits mais le rapport entre les niches écologiques (modes d'insertion dans le milieu) et les variations environnementales se prête à des séries de reconstitutions plus fines. En puissance, du moins, car nous manquons d'éléments sur la répartition spatiale des chimpanzés plus paisibles de Côte d'Ivoire, par exemple, voire sur l'agressivité peutêtre plus intense dans les relations inter-groupes des chimpanzés moins connus de savane (Moore 1996) ${ }^{7}$. Les multiples singes non omnivores se livrant peu ou prou à l'infanticide mais pas au cannibalisme (à commencer par l'entelle) posent une autre question: la tendance prédatrice du chimpanzé ajoute-t-elle quelque chose à l'agression, ou bien est-elle accessoire et subalterne? À l'étage du fait, les réponses à ces problèmes déterminent largement un enrichissement des conjectures sur les chasseurs du paléolithique inférieur, généralement marqués par une forte dispersion, mais qui, en certaines régions et certaines époques, ont sûrement connu des phases de relative concentration (ne serait-ce que saisonnières) où les relations intergroupes prenaient une tournure particulière.

Sans doute les perspectives à long terme obligent-elles à subodorer des combinaisons et des corrélations entre l'évolution du psychisme et la figuration d'une " place dans la nature ». Toutefois, de telles conciliations thématiques ne s'élaborent pas selon la même procédure selon que l'on entre dans le carrefour (et que l'on en sort) par l'une ou l'autre route. Ainsi, parler de "l'évolution du psychisme », c'est suggérer une dynamique, à l'inverse de la « place dans la nature ». Mais si l'on remplace cette vieille notion statique, issue $\mathrm{du}$ « darwinisme social », par le concept scientifiquement contrôlable de «niche écologique », on supprime une barrière mentale: l'exploitation du milieu par une espèce intervient elle aussi activement sur les dispositions du psychisme.

En dépit de ce que les sciences de la vie et de l'homme nous donnent à voir depuis plus d'un siècle, la comparaison n'est pas censée s'amollir sur le terrain vague de l'interdisciplinarité alors qu'elle conserverait davantage de rigueur dans le jardin mieux rangé (ou plus attentivement surveillé) d'un secteur scientifique rôdé. Aucun motif ne l'autorise à se contenter sur une vaste étendue d'un amoncellement de similitudes ponctuelles qu'elle s'interdirait à petite échelle: contrairement à l'analogie, qui se complaît dans des rapprochements subjectifs, le travail comparatif progresse toujours grâce aux contrastes et se nourrit de chaque hiatus. Le surgissement de la différence lui offre un ancrage sur le réel. 
Entre les actes de la guenon de Gombe tenue ensuite à l'écart par certains individus dominants du groupe et les attaques perpétrées par certains mâles de Mahale, il y a des ressemblances et des dissemblances mais le seul fait brut susceptible d'informer l'anthropologie à partir de leur addition est que l'appétit cannibale n'a rien de simple à ce niveau embryonnaire de la culture: il dépasse sans équivoque une réponse automatique à la faim, ou un banal réflexe de prédateur, puisque tous les individus n'y succombent pas, que certains s'y opposent et que, dans l'un et l'autre cas, les cibles ont une identité sociale strictement définie. Quant au parachutage « du » cannibalisme chimpanzé sur ceux de la Nouvelle-Guinée, de l'Amazonie ou des Néandertaliens, il nous instruit seulement sur la survivance énigmatique de l'évolutionnisme archaïque bien qu'il ait depuis longtemps démontré son inaptitude. En une époque où les darwinistes d'avant-garde nous enseignent vertueusement que "nous ne descendons pas du chimpanzé », il ne coûterait pas grand-chose de rappeler que la proposition vaut autant pour les «sociétés primitives». Faute de quoi, par le biais de comportements horriblement émoustillants rejetés par l'humanisme bien senti et « conservées » par quelques cultures exotiques, la discussion sera discrètement compartimentée: d'un côté, le rapport singes/primitifs pour les biologistes, de l'autre le rapport primitifs/civilisés momentanément abandonné aux sociologues.

\section{L'évidence refoulée: la stipulation du cadre de référence}

Laspiration idéologique de la sociobiologie et la fascination du cannibalisme accroissent les manifestations d'une forme de dérapage méthodologique qu'il est facile de constater à travers l'ensemble des dialogues entre sciences naturelles et sciences sociales. La réflexion interdisciplinaire refuse d'obéir à une obligation que chaque discipline veille pour sa part à faire respecter en son domaine: le maintien de l'argumentation dans un cadre de référence explicite ou déductible, mais de toute façon contrôlable. Par cadre de référence, on entend ici la délimitation d'un espace et d'un temps propres à la résolution d'un problème énoncé.

C'est certainement une évidence, mais, ici, une évidence évidemment évacuée. L'extension d'une réponse hors du cadre de référence où la question avait un sens avéré est une maladie chronique de l'interdisciplinarité, laquelle n'imagine toujours pas que la constitution d'un cadre commun puisse être le préalable ou le début de son activité. Il suffit d'imaginer un match âprement disputé entre des golfeurs et des rugbymen: pour avoir une chance de deviner à quoi ils jouent, il faudrait déjà savoir où ils jouent. Souvent, les acteurs profitent cyniquement de ce que leurs adversaires ne respectent pas l'exigence de délimitation pour ne pas la respecter non plus. Peut-on alors formuler avec un minimum de clarté une règle de bonne conduite? 
Le point de départ existe sans doute dans la controverse sur les Aztèques. Lambition fonctionnaliste que Sahlins raillait chez Harner et Harris n'a rien de scientifiquement condamnable. Quoi de plus légitime, pour un chercheur que d'espérer découvrir des causes à des faits réguliers? En revanche, avant de soutenir l'existence d'une fonction régissant un fait social ou avant de la contrecarrer, les protagonistes, au risque de rendre la polémique moins attractive, auraient dû stipuler successivement les conditions du cadre correct de l'élaboration et celles du cadre convenable de la réfutation. Une hypothèse de type fonctionnelle, telle que celle proposée par Harner réclame nécessairement:

a) un cadre de référence initial qui met en relief l'existence d'une possible relation (tel que le profit retiré par l'aristocratie aztèque de l'anthropophagie guerrière);

b) un cadre de référence en amont, génétique au sens premier, qui envisage les paramètres favorables et défavorables à l'installation pratique de cette relation;

c) un cadre de référence en aval, systémique, qui traite de ses moyens de persistance, de pérennisation et d'autorégulation. La pondération de la relation face aux autres influences agissant dans le milieu social ne saurait évidemment être déduite d'office du deuxième cadre, car la création d'un phénomène et sa perpétuation ne répondent pas au même jeu logique.

Dans une seconde phase, le renfort essentiel du traitement comparatif (autres milieux, autres peuples, autres époques) réclame que ces trois niveaux soient mis à l'œuvre sans être jamais substitués l'un à l'autre: on verra très bientôt que ceci implique l'installation préalable d'une autre tripartition. Harner posait un cadre initial, puis raisonnait comme si l'ampleur de l'anthropophagie aztèque suffisait à indiquer l'existence des deux suivants. Et, une fois décelé, il est aisément constatable que cet expédient discret ravage la quasi-totalité des assertions sur la fonction éminente d'un cannibalisme.

Le mécanisme à partir duquel les idéologies occidentales pénètrent et contrôlent les interprétations du cannibalisme se comprend dans une image paradoxale: ce thème se dresse comme un pont permanent sur la mouvante frontière nature/culture, une passerelle immuable entre deux espaces en perpétuelle transformation. Durant le dernier siècle, malgré les vœux vibrants périodiquement prononcés en faveur d'une installation de l'interdisciplinarité, aucun secteur scientifique ne s'est solidement construit en s'établissant simultanément et avec la même vigueur du côté de la nature et du côté de la culture. Peu importe, sur ce point précis, que l'on soit ou non convaincu de l'existence objective de cette ligne de démarcation: la science qui y croit n'a jamais réussi à connecter ce qu'elle prétendait distinguer, et la science qui n'y croit pas n'est pas davantage parvenue à unir ce qu'elle se refusait à séparer.

Cependant, l'ambition fonctionnaliste n'est à son tour que le cadre restreint et théoriquement orienté d'une volonté scientifique plus large à laquelle doit s'attacher une expression synthétique d'un modus operandi. valable 
pour les différentes écoles. Proposons donc à partir du système précédent une règle « garde-fou » dont le champ d'application couvrirait la majorité des conjectures enjambant la démarcation nature/culture, dans un sens ou dans l'autre. Ne serait-ce que pour attirer l'attention sur la difficulté à résoudre d'urgence. Nous soutenons que toute recherche sur un hypothétique type d'action, ou d'interaction, devrait s'obliger à respecter une distinction immédiate et permanente entre trois cadres de référence élémentaires:

a) celui de lévénement, qu'il soit alors question d'une observation effective ou d'une reconstitution a posteriori;

b) celui du fait régulier, où l'on examine la répétition de l'événement dans un contexte délimité, puis dans une série de contextes comparables;

c) celui de la tendance, enfin, qui modélise la probabilité de l'événement en fonction d'une délicate pondération des circonstances incitatrices et des facteurs inhibiteurs. Zone scabreuse parce qu'attirante pour les velléités d'extensions incontrôlées.

La reconnaissance signalée plus haut à propos de Harner des trois cadres nécessaires à une explication fonctionnelle ou fonctionnaliste se résume maintenant à une modalité particulière de la règle générale, car la définition d'un cadre de référence ne requiert pas seulement un repérage de l'entité spatiale et temporelle dans laquelle on s'engage à résoudre une énigme, mais également l'analyse formelle de la question posée (faute de quoi il serait évidemment impossible d'évaluer la pertinence du cadre de référence choisi au regard du but à atteindre). Afin de démasquer les analogies abusives, les extrapolations non fondées et les dérives purement intuitives, le principe élargi est en effet énoncé de manière à « coiffer » des perspectives théoriques opposées et devrait permettre aussi bien à un émule de Marshall Sahlins de montrer les failles dans une argumentation réductrice qu'à un disciple de Marvin Harris de critiquer les lacunes d'une interprétation culturaliste.

La tendance qui s'emploie à absorber directement un événement sans qu'on puisse l'apercevoir sur les divers degrés accessibles du fait, ou l'événement aussitôt catalogué comme la manifestation d'une tendance, induisent deux enlisements symétriques dans la spéculation pure : l'extension ou la régression analogiques n'y sont finalement stimulées que par la croyance même qu'elles veulent renforcer. Tout simplement parce que la cohérence interne d'un événement peut se révéler une anomalie au regard de la tendance et que celle-ci peut correspondre fortuitement à un événement, c'est-à-dire de façon inactive et insignifiante (cf., plus haut, les meurtres des chimpanzés). Dans tous les cas, une rationalité conçue à une certaine échelle est arbitrairement transférée à un autre étage, soit pour « prouver » sa présence à cet étage, soit pour confirmer son existence à l'échelle initiale.

Au cours de la dernière décennie, il a été de bon ton d'ironiser sur les espoirs déçus et les promesses non tenues de l'interdisciplinarité, comme si la voie elle-même s'était révélée impraticable ou illusoire. Il faut prendre conscience que nous avons voulu atteindre les synthèses 
et les « interfaces » en pariant naïvement sur des clefs universelles ouvrant indifféremment toutes les portes du microcosme au macrocosme: cela est allé des lois métaphysiques telles que le gène égoïste jusqu'aux modèles métascientifiques comme l'analyse systémique, le paradigme de la complexité, etc.

Nous avons ainsi dépensé beaucoup d'énergie à nous ruer sur des trompe-l'œil. Et la recherche appliquée en a pâti davantage encore que la recherche fondamentale, ne serait-ce qu'en se laissant séduire par des passe-partout illusoires: des modèles acceptables par tous qui suffiraient à garantir des coopérations enfin paisibles. Alors, au sortir de la nuée des idées interchangeables et avant de saisir la dérision comme une ultime et irréductible sagesse à géométrie variable - car elle corrode, elle aussi, toutes les échelles -, n'est-il pas temps de pressentir l'interdisciplinarité dans un surcroît d'auto-discipline?

Est-il légitime de transformer les leçons d'un événement anecdotique en un fait d'évolution, ou de transférer la conclusion d'une observation ponctuelle dans la longue durée d'un vaste monde? Sûrement pas. Pourtant, ces extensions purement analogiques sont fréquentes en sciences naturelles et en sciences humaines et cela d'autant plus que la proposition est « téléportée » des unes vers les autres. Le cannibalisme, de par la phobie qu'il inspire à notre civilisation, augmente les risques de dérapages théoriques et il offre ainsi une sorte de loupe méthodologique devant ce problème.

\section{$\&$}

Tout ce qui tourne autour du rapport nature/culture, de la frontière animal/ homme, ou de la connexion biologique/social se caractérise comme une zone de stagnation théorique: non seulement les antagonismes philosophiques semblent s'y cristalliser désespérément, mais ils paraissent euxmêmes produire une immobilisation des tendances intellectuelles et forger des structures de pensée capable de résister à l'afflux des informations pourtant inattendues que livre régulièrement l'observation scientifique. Les cibles de la recherche ont beau avoir beaucoup changé depuis le milieu du XIX e siècle, ainsi que les supports de l'argumentation, force est de constater deux faits troublants au sujet des discussions majeures qui se sont nouées sur ces sujets: elles s'agglutinent irrésistiblement les unes aux autres jusqu'à ce que leur confusion totale fasse figure d'évidence et elles se répètent périodiquement sous des rénovations sémantiques superficielles. Ces deux volets - confusion et répétition - ne sont ici distingués que par commodité, car un seul ressort autorise l'extension thématique et la reproduction historique: une liberté tacitement et collectivement accordée au glissement analogique des raisonnements.

Est-ce une cause ou un effet? Cette mystérieuse plasticité des dissensions 
théoriques ne se borne pas à garantir la pérennité des grandes alternatives philosophiques, elle permet également la reconduction de contradictions que les institutions rendent plus palpables, à commencer par la tension « traditionnelle » qui sévit entre les tenants des sciences de la vie et ceux des sciences sociales. Par exemple, côté « confusion», la synonymie souvent affirmée ex nihilo entre les rapports nature/culture et inné/acquis facilitent d'emblée un ordre de marche de toute la biologie à la rencontre des phénomènes sociaux: peu importerait que l'explication fondatrice adressée à la sociologie soit d'ordre génétique, éthologique ou écologique (malgré les profondes divergences épistémologiques qui séparent ces secteurs), du moment qu'elle puisse se réclamer d'une autorité de principe des sciences de la vie sur les sciences sociales, affreusement «molles » comme chacun sait. Et, du côté de la « structure réitérative des grandes idéologies para-scientifiques » (Tort 1983), on se contentera de rappeler le réaménagement complet du vieux darwinisme social en sociobiologie, moyennant un remplacement du support imaginé pour l'évolution de l'altruisme: l'hérédité lamarckienne des caractères acquis a cédé la place aux spéculations du gène égoïste. Là aussi, la résurrection a été secondée par une réorganisation pluridisciplinaire du réductionnisme (absorption supposée de l'éthologie par la zone d'influence de la dynamique des populations, assujettissement de fait de la socio-écologie animale à une « écologie comportementale » créée pour l'occasion): une restriction terrible des possibilités de dialogue avec les anthropologues qui ne pouvaient accepter que la discussion soit subordonnée à la reconnaissance d'une loi universelle jaillie du monde moléculaire. 


\section{NOTES}

1. Seules les femelles viennent d'œufs fécondés et sont diploïdes, alors que les mâles sont haploïdes et ont donc deux fois moins de chromosomes.

2. Nous ne pouvons donner ici les titres (beaucoup trop nombreux) des publications favorables à ces explications inconséquentes: citons donc seulement à l'origine William D. Hamilton (1964) et Edward O. Wilson (1975) pour les insectes " eusociaux », et Sarah B. Hrdy (1984) pour l'entelle.

3. Dont un pour signaler que des pesticides et autres produits non naturels pourraient rendre la viande impropre à la consommation et qu'une agence gouvernementale devrait établir des standards à ce sujet (Posinsky 1971). Sauf méprise de notre part, il s'agit d'un rarissime cas d'humour en anthropologie, toutes nations confondues.

4. Bien que cette mini-polémique se soit cantonnée dans un cercle relativement fermé, soulignons que l'anthropologie biologique l'a suivie de près et les dernières évaluations citées ont figuré au premier degré comme indications de base dans des ouvrages de référence sur les moeurs alimentaires de notre espèce (Farb \& Armelagos 1979).

5. Mariko Hiraiwa-Hasegawa (1992) en compte 14 mais nous y avons ajouté un cas (daté de 1979) relaté par Jane Goodall (1986) et nous avons retiré deux faits (l'un à Gombe, l'autre à Mahale) où l'enfant a été tué mais non mangé. D'autres faits de cette sorte ont eu lieu ultérieurement, mais, ne disposant pas d'un inventaire postérieur à celui d'Hiraiwa-Hasegawa, nous préférons nous en tenir à ce corpus qui semble à peu près complet jusqu'en 1989.

6. Une centaine de kilomètres, environ, les séparent à vol d'oiseau.

7. Encore ne faut-il pas, là non plus, en tirer des conclusions hâtives sur la violence au Paléolithique ancien avant d'avoir obtenu quelques évaluations sur la situation démographique de ces populations.

8. Bien qu'il ne soit pas souhaitable de s'y attarder ici, il est clair que cette proposition recoupe le double concept de «tendance et fait », associé à l'œuvre d'André Leroi-Gourhan. Plus qu'en ethnologie, cependant, c'est dans le patient travail de l'histoire et de l'archéologie des techniques que la différence de traitement entre le cadre de l'événement (ou, en l'occurrence, le document représenté par un objet) et celui du fait (ensembles et sous-ensembles) s'impose avec le plus de netteté (Sophie Desrosiers, communication personnelle). 


\section{RÉFÉRENCES}

Dawkins, Richard, 1989, L'Horloger aveugle. Paris, Robert Laffont.

Dornstreich, M. D. \& G. E. B. Morren, 1974, « Does New Guinea Cannibalism Have Nutritional Value? », Human Ecology 2(1) : 1-12.

Farb, Peter \& George Armelagos, 1985, Anthropologie des coutumes alimentaires. Paris, Denoël.

Garn, Stanley, 1979, "The noneconomic nature of eating people », American Anthropologist 81 : 902-903.

Garn, Stanley \& Walter D. Block, 1970, « The limited nutritional value of cannibalism », American Anthropologist 72: 106.

Goodall, Jane, 1986, The Chimpanzees of Gombe. Patterns of Behavior. Boston, The Belknap Press of Harvard University Press

Guille-Escuret, Georges, 1994, Le Décalage humain. Le fait social dans l'évolution. Paris, Kimé.

Guille-Escuret, Georges, 1996, "Corrélations statistiques et évolutionnismes modernes », in Patrick Tort (ed.), Dictionnaire du darwinisme et de l'évolution. Paris, P.U.F : 702-705.

Guille-Escuret, Georges, 1997a, "L'évolution, "toutes choses égales par ailleurs" » : pour une épistémologie interne du darwinisme », in Patrick Tort (ed.), Pour Darwin. Paris, P.U.F. : 785-803.

Guille-Escuret, Georges, 1997b, «Le biologisme revigoré: vie/société, nature/culture, évolution/histoire », Diogène 180: Le gène est-il l'avenir de l'homme?: 5-21.

Harner, Michael, 1977, « The ecological basis for Aztec sacrifice », American ethnologist 4: 117-135.

Harris, Marvin, 1979, Cannibales et monarques. Paris, Flammarion.

Hiraiwa-Hasegawa, Mariko, 1992, "Cannibalism among non-human primates », in Mark A. Elgar \& Bernard J. Crespi, 1992, Cannibalism. Ecology and Evolution among Diverse Taxa. Oxford, Oxford University Press: 323-338.

Hrdy, Sarah Blaffer, 1984, Des guenons et des femmes. Essai de sociobiologie. Paris, Tierce.

Hublin, Jean-Jacques, 1982, «Cannibalisme et archéologies », Histoire et Archéologie 66: 24-27.

Joulian, Frédéric, 1995, « Mise en évidence de différences traditionnelles dans le cassage des noix chez le chimpanzé (Pan troglodytes) de la Côte d'Ivoire, implications paléoanthropologiques », Journal des Africanistes 65 (2): 57-77.

McGrew, William C., 1992, Chimpanzee Material Culture. Implications for Human Evolution. Cambridge, Cambridge University Press.

Moore, Jim, 1996, «Savanna chimpanzees, referential models and the last common ancestor », in William C. McGrew, Linda F. Marchant et Toshisada Nishida (eds), Great Apes Societies. Cambridge, Cambridge University Press : 275-292.

Norikoshi, Kohshi, 1982, « One Observed Case of Cannibalism Among Wild Chimpanzees of the Mahale Mountains », Primates 23 (1): 66-74.

Posinsky, S. H., 1971, «Cannibalism », American Anthropologist 73: 269.

Randall, Mark E., 1971, "Comment on "The Limited Nutritional Value of Cannibalism" ", American Anthropologist 73: 269 .

Rappaport, Roy A., 1968, Pigs for the Ancestors. Ritual in the Ecology of a New Guinea People. New Haven, Yale University Press.

Sahlins, Marshall, 1979, «Culture, protéines, profit », Libre 5: 105-128.

Soltis, Joseph, Boyd, Robert, \& Richerson, Peter J., 1995, « Can Group-Functional Behaviors Evolve by Cultural Group Selection? An Empirical Test », Current Anthropology 36: 473-494.

Thierry, Bernard, 1997, «Adaptation et auto-organisation dans les sociétés de primates », Diogène 180: Le gène est-il l'avenir de l'homme? : 37-66.

Tort, Patrick, 1983, La Pensée hiérarchique et l'évolution. Paris, Aubier. 
Tuttle, Russell H.,1986, Apes of the World. Their Social Behavior, Communication, Mentality and Ecology. Park Ridges (New Jersey), Noyes Publications.

Vayda, Andrew P., 1970, « On the nutritional value of cannibalism », American Anthropologist 72: 14621463.

Walens, Stanley, 1971, « Pigs, Proteins, and People-Eaters », American Anthropologist, 73: 269-270.

Wilson, Edward O., 1975, Sociobiology: The New Synthesis. Cambridge (Mass.), The Belknap press of Harvard University Press. 


\section{RÉSUMÉ}

Le syndrome Micromégas. L'expérience historique montre que, régulièrement, les débats théoriques sur le rapport nature/culture s'immobilisent en des antagonismes bipolaires et que ceux-ci se superposent comme s'il n'y avait en définitive qu'un seul duel avec de multiples facettes. Nous sommes tellement habitués à cette situation que nous finissons par la ressentir comme une évidence implacable. Au mieux, nous l'interprétons comme l'issue d'une confrontation idéologique permanente. Cependant, on oublie alors qu'une confrontation de cette sorte ne peut s'installer qu'en obtenant un minimum de garanties de part et d'autre: il faut que les ennemis soient tacitement d'accord sur certaines « règles du jeu ». Ou, plus exactement, sur les façons d'enfreindre celles qui devraient prévaloir dans le jeu scientifique.

La plus importante de ces règles, sans doute, consiste à accepter que les questions se déplacent sans contrôle d'une échelle de réalité à l'autre, d'un niveau de structuration à l'autre: du microcosme au macrocosme. Or, chaque interrogation appelle un cadre de référence spatial et temporel qui est nécessaire au contrôle de sa teneur. Et elle se transforme au fur et à mesure que l'on modifie ou que l'on déplace ce cadre.

À travers quelques débats fameux sur les « causes » du cannibalisme, cet article montre comment la discussion scientifique demeure muselée par un manque de surveillance sur ce plan et comment elle est artificiellement immobilisée par le refus général de distinguer des cadres de référence différents pour des questions s'attachant à des aspects incommensurables de la causalité.

\section{ABSTRACT}

The Micromegas Syndrome. Historical experience shows that theoretical debates on the relationship nature/ culture regularly come to a halt along bipolar antagonisms and these are superposed as if there were in reality a single duel with multiple aspects. We are so used to this situation that we end up feeling it is absolutely obvious. At best, we interpret it as the result of a permanent ideological confrontation. However, we then forget that such a confrontation can be established only by obtaining minimum guarantees from one side or the other: enemies have to tacitly agree on certain « rules of the game » or more precisely, on how to break the meaningful rules in the scientific game.

The most important of these rules is no doubt accepting that questions shift independently from one scale of reality to another, from one design level to another: from microcosm to macrocosm. Or, each questioning calls for a spatial and temporal reference frame that is necessary to control its terms. And it transforms itself according to whether it is modified or shifted in this frame.

Through some well know debates on the « causes » of cannibalism, this article shows how scientific discussion remains bound by a lack of survey on this level and how it is artificially immobilized by the general refusal to distinguish different reference frames for questions bound to the immeasurable aspects of causality.

\section{MOTS CLÉS}

Aztèque, cannibalisme, déterminisme, épistémologie, Nouvelle-Guinée, primate, sociobiologie.

\section{KEYWORDS}

Aztec, cannibalism, determinism, epistemology, New Guinea, primate, sociobiology. 